but the new Sivalik finds do at least document the presence of tree shrews of modern aspect in India some 10 million years or so ago, perhaps contemporaneously with such undoubted prosimian primates from the Sivaliks as Indraloris (Lewis Am.J.Sci. 26 134; 1933; Tattersall Postilla (Yale) 123, 1; 1968).

\section{Origin of the Galilean satellites and Jovian rings}

\section{from David W. Hughes}

REGULAR satellite and ring systems seem to be a common feature of the major planets. These systems are coplanar, the orbits are circular and also the ratio between the orbital radius of a satellite and that of the one immediately interior is a constant, about 1.65 in the case of Jupiter.

The formation of satellites as the planets condensed has often been linked with the formation of the planets as the Sun condensed, and this process has been reinvestigated by Prentice and ter Haar in a recent edition of Nature $(280,301 ; 1979)$. These authors return to the hypothesis originally advanced by Laplace in 1796. In the beginning, according to Laplace, the solar and planetary material was in the form of a huge spinning gaseous globe with a diameter larger than that of Neptune's orbit. As this great globe cooled it contracted, spinning faster and faster due to the conservation of angular momentum. A stage was eventually reached when the equatorial centrifugal force became larger than the gravitational force and a ring of matter was left behind, suspended in a circular orbit in the equatorial plane. Due to this mass and angular momentum loss, the proto-Sun could contract a little further, but it soon became unstable again and shed another ring. This process was repeated until the Sun reached its present size.

Now it must be remembered that the present mass of the Solar System is only a very small fraction of the mass of the rings, simply because most of the planets consist of material that is quite rare by normal cosmic standards. Each planet is probably the condensation remnant of a ring of about a thousand times the Earth's mass. Also the spinning gaseous globe was probably strongly turbulent. By considering T. Tauri stars Prentice (Astr. Astrophys. 27, 237; 1973) concluded that the convective velocities in the globe are supersonic and that the turbulent stresses set up by these convective movements are of the order of 30-100 times the normal gas pressure just below the surface. Two things result from this. First, the globe becomes very centrally condensed which obviously leads to a low moment of inertia. Second, a dense shell of non-turbulent gas develops at the surface, this evolving into an equatorial belt as the cloud contracts. It is this belt which is left behind when centrifugal force exceeds gravitational force, and it can be shown that the ring-shedding process repeats itself at a sequence of orbital radii $R_{n}$ which satisfy

$$
R_{n} / R_{n+1}=\left(1+m / M_{\mathrm{e}}\right)^{2} .
$$

In this equation $m$ is the mass of the ring, $M_{\mathrm{e}}$ is the mass of the gaseous globe that has just lost the ring and $f$ equals $I_{\mathrm{e}} / M_{\mathrm{e}} R_{\mathrm{e}}{ }^{2}$ where $I_{\mathrm{e}}$ and $R_{\mathrm{e}}$ are the moment of inertia and radius of the globe.

For the specific case of the Galilean satellites of Jupiter Prentice and ter Haar set $M_{\mathrm{e}}$ to $1.9 \times 10^{30} \mathrm{~g}$ the present mass of Jupiter, $f$ to 0.02 and $R_{n} / R_{n+l}$ to 1.65 . This immediately tells us that the mass of each Jovian ring was about $1.1 \times 10^{28} \mathrm{~g}$. If this mass was of solar composition it would have two medium-temperature $\left(\sim 0^{\circ} \mathrm{C}\right)$ solid components. The rocky one would have a mass of about $5 \times 10^{25} \mathrm{~g}$ and the water ice and rock mixture component would have a mass of about $12 \times 10^{25} \mathrm{~g}$. The globe is warming up as it condenses so each detached ring will have a different temperature. The authors calculate that the temperatures at detachment for Io, Europa, Ganymede and Callisto are 533, 338,220 and $133 \mathrm{~K}$ respectively. It is this temperature difference which causes the chemical variation. Io and Europa with masses of $9 \times 10^{25}$ and $5 \times 10^{25} \mathrm{~g}$, respectively, have little ice, the hightemperature Io condensation having iron in the form of $\mathrm{FeS}$ while for Europa, $\mathrm{Fe}$ appears as $\mathrm{FeO}$. The heat released as the ring condenses to form a satellite and as the satellite compacts causes $\mathrm{S}$ and $\mathrm{H}_{2} \mathrm{O}$ to leak out, leaving sulphur deposits on Io and icy plains on Europa. Ganymede and Callisto condensed at a temperature below the freezing point of water and therefore consist of dirty ice.

By extending this theory, the authors predict that the contracting gaseous globe can shed one or two more rings interior to Io. One has a radius of $2.5 R_{\mathrm{p}}$ and the other $4 R_{\mathrm{p}}$ where $R_{\mathrm{p}}$ is the present Jovian polar radius, $6.677 \times 10^{9} \mathrm{~cm}$. Amalthea is near the first of these but is spectrally very close to a C-type asteroid and has probably been captured by Jupiter at a later date. These interior gaseous rings probably did not condense to form satellites because there was too little time for the rocks to aggregate into a single body before the gaseous component of the rings dispersed, taking with it the finer dust material. Also the increasing luminosity of proto-Jupiter probably frustrated the settling out process.

Ring shedding cannot proceed indefinitely. The rate of release of gravitational potential energy slows down between $3 R_{\mathrm{p}}$ and 1.5 $R_{\mathrm{p}}$ in the case of Jupiter. This results in a decrease in the surface temperature and in the supersonic

David $W$. Hughes is a Lecturer in the Department of Physics, University of Sheffield. turbulence. $R_{n} / R_{n+1}$ drops to 1.02 , leaving the rings so closely spaced that they essentially merge together to form a continuous disk. The disk does not coalesce into a few single bodies because of its differential rotation. The gaseous component disperses and orbital resonance subsequently changes its structure. It is these resonances which may account for the sharp ring edge seen at $1.9 R_{\mathrm{p}}$ by the Voyager I and II spacecraft.

No theory in cosmogony is without its rival and the origin of the Jovian rings is no exception. Smoluchowski wrote in the following issue of Nature $(280,377 ; 1979)$ that the rings were probably produced by the tidally induced fragmentation of a nonspherical, mechanically weak carbonaceous body. This disruption is thought to have occurred fairly recently. The solid surface area of the rings has been estimated to be about $20,000 \mathrm{~km}^{2}$, this value coming from a comparison between the decrease produced in the charged particle flux by the rings and that caused by Amalthea, both these effects being observed by Pioneer 11. Bombardment by the intense flux of protons in the Jovian Van Allen belts erodes particles. Smoluchowski calculated that ice was eroded at between 50 and $100 \dot{\mathrm{A}} \mathrm{yr}^{-1}$ and silicate particles at 2-3 $\AA \mathrm{yr}^{-1}$. Many small grains are thus eliminated on the time scale of a million years and the Jovian rings are not expected to have a lifetime much longer than this.

\section{Normal fluid density of superfluid ${ }^{3} \mathbf{H e}-\mathrm{B}$}

\section{from P.V.E. McClintock}

NEW high precision measurements of the normal fluid density of liquid ${ }^{3} \mathrm{He}-\mathrm{B}$ have yielded some surprises. In particular, it seems that the so-called 'stripped' normal fluid density is independent of pressure, quite contrary to expectation. The experiments were carried out by Archie, Alvesalo, Reppy and Richardson of Cornell University and are reported in a recent issue of Physical Review Letters (43, $139 ; 1979)$.

The hydrodynamic properties of the B-phase of ${ }^{3} \mathrm{He}$ are relatively simple in that the liquid can conveniently be described in terms of a two-fluid model, qualitatively very similar to that which has been used in relation to superfluid ${ }^{4} \mathrm{He}$ for many years. Thus, the liquid is envisaged as being composed of two quite separate but completely interpenetrating fluids: a normal fluid component whose properties are similar to those of conventional liquids; and a superfluid component able to indulge in dissipation-free flow, carrying no entropy, and responsible for much of the seemingly bizarre behaviour exhibited by 